\title{
Overdensity of SubMillimiter Galaxies in the GJ526 Field mapped with the NIKA2 Camera
}

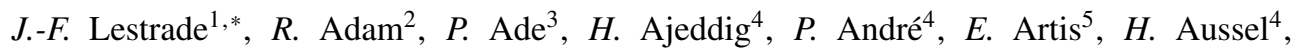
A. Beelen ${ }^{6}$, A. Benoît ${ }^{7}, S$. Berta $^{8}, L$. Bing $^{9}, O$. Bourrion $^{5}, M$. Calvo $^{7}, A$. Catalano $^{5}$, A. Coulais ${ }^{1}, M$. De Petris ${ }^{10}, F .-X$. Désert ${ }^{11}, S$. Doyle ${ }^{3}$, E. F. C. Driessen ${ }^{8}, A$. Gomez $^{12}$, J. Goupy ${ }^{7}, F$. Kéruzoré ${ }^{5}, C$. Kramer ${ }^{13}$, B. Ladjelate ${ }^{13}, G$. Lagache $^{9}, S$. Leclercq ${ }^{8}$, J.F. Macías-Pérez ${ }^{5}, A$. Maury ${ }^{4}, P$. Mauskopf ${ }^{3,14}, F$. Mayet $^{5}, A$. Monfardini ${ }^{7}, M$. MuñozEcheverría $^{5}, L$. Perotto ${ }^{5}, G$. Pisano ${ }^{3}, N$. Ponthieu ${ }^{11}, V$. Revéret $^{4}, A . J$. Rigby $^{3}, A$. Ritacco $^{6,15}$, $C$. Romero ${ }^{16}, H$. Roussel ${ }^{17}, F$. Ruppin ${ }^{18}, K$. Schuster ${ }^{8}, S$. Shu $^{19}, A$. Sievers ${ }^{13}, C$. Tucker ${ }^{3}$, and R. Zylka ${ }^{8}$

${ }^{1}$ LERMA, Observatoire de Paris, PSL Research University, CNRS, Sorbonne Université, UPMC, 75014 Paris, France

${ }^{2}$ LLR (Laboratoire Leprince-Ringuet), CNRS, École Polytechnique, Institut Polytechnique de Paris, Palaiseau, France

${ }^{3}$ School of Physics and Astronomy, Cardiff University, Queen's Buildings, The Parade, Cardiff, CF24 3AA, UK

${ }^{4}$ AIM, CEA, CNRS, Université Paris-Saclay, Université Paris Diderot, Sorbonne Paris Cité, 91191 Gifsur-Yvette, France

${ }^{5}$ Univ. Grenoble Alpes, CNRS, Grenoble INP, LPSC-IN2P3, 53, avenue des Martyrs, 38000 Grenoble, France

${ }^{6}$ Institut d'Astrophysique Spatiale (IAS), CNRS, Université Paris Sud, Orsay, France

${ }^{7}$ Institut Néel, CNRS, Université Grenoble Alpes, France

${ }^{8}$ Institut de RadioAstronomie Millimétrique (IRAM), Grenoble, France

${ }^{9}$ Aix Marseille Univ, CNRS, CNES, LAM (Laboratoire d'Astrophysique de Marseille), Marseille, France

${ }^{10}$ Dipartimento di Fisica, Sapienza Università di Roma, Piazzale Aldo Moro 5, I-00185 Roma, Italy

${ }^{11}$ Univ. Grenoble Alpes, CNRS, IPAG, 38000 Grenoble, France

${ }^{12}$ Centro de Astrobiología (CSIC-INTA), Torrejón de Ardoz, 28850 Madrid, Spain

${ }^{13}$ Instituto de Radioastronomía Milimétrica (IRAM), Granada, Spain

${ }^{14}$ School of Earth and Space Exploration and Department of Physics, Arizona State University, Tempe, AZ 85287, USA

${ }^{15}$ Laboratoire de Physique de l'École Normale Supérieure, ENS, PSL Research University, CNRS, Sorbonne Université, Université de Paris, 75005 Paris, France

${ }^{16}$ Department of Physics and Astronomy, University of Pennsylvania, 209 South 33rd Street, Philadelphia, PA, 19104, USA

${ }^{17}$ Institut d'Astrophysique de Paris, CNRS (UMR7095), 98 bis boulevard Arago, 75014 Paris, France

${ }^{18}$ Kavli Institute for Astrophysics and Space Research, Massachusetts Institute of Technology, Cambridge, MA 02139, USA

${ }^{19}$ Caltech, Pasadena, CA 91125, USA

Abstract. Using the NIKA2 dual band millimeter camera installed on the IRAM30m telescope, we have mapped a relatively large field $\left(\sim 70 \operatorname{arcmin}^{2}\right)$ in the direction of the star GJ526 to investigate the nature of the sources found

*e-mail: jean-francois.lestrade@obspm.fr 
with the MAMBO camera at $1.2 \mathrm{~mm}$ ten years earlier. We have found that they must be dust-obscured galaxies (SMGs) in the background beyond the star. The new NIKA2 map at $1.15 \mathrm{~mm}$ reveals additional sources and, in fact, an overdensity of SMGs predominantly distributed along a filament-like structure in projection on the sky across the whole observed field. We speculate this might be a cosmic filament at high redshift as revealed in cosmological hydrodynamical simulations. Measurement of spectroscopic redshifts of the SMGs in the candidate filament is required now for a definitive confirmation of the nature of the structure.

\section{Introduction}

Surveys at submillimeter and millimeter-wavelengths have revealed a dust-obscured population of galaxies at high redshift $[1,2]$. These massive SubMillimeter Galaxies (SMGs) are forming stars at prodigious rates, sometimes exceeding $1000 M_{\odot} / \mathrm{yr}$, with correspondingly bright far infrared luminosities larger than $10^{12} L_{\odot}$ [3]. SMGs are enshrouded in dust and so have only faint or undetectable optical counterparts but contribute to the far infrared cosmic background emitting approximately half of all of the light in the universe $[4,5]$. Theories predict that galaxy formation preferentially occurs along large-scale dark matter filamentary or sheetlike overdense regions that are related to initial fluctuations of the density field in the primordial universe [6] and formed the cosmic web as depicted by cosmological hydrodynamical simulations. It is thought that star formation and galaxy growth at an early stage of cosmic evolution are critically tied to this network of dark matter filaments. A large fraction of the neutral hydrogen from the inter galactic medium falls into them and constantly replenishes the gas reservoirs of galaxies through cold gas accretion ; the cold gas streams along the filaments, survives the shocks at the virial radii of the dark matter halos and infalls onto the galaxies to fuel their star formation and growth [7, 8]. However, definitive observational confirmation of cold gas accretion along filaments of the cosmic web is actively sought.

A region of the sky several hundreds of arcseconds in size corresponds to an area of several cMpc at high reshift (scaling factor cMpc/" does not depends strongly on $z$ at high $z$ in standard cosmology) that can be mapped efficiently at millimeter wavelengths using a modern camera such as NIKA2 on the IRAM30m radiotelescope [9-14]. SMGs detected in such a field must be embedded in filaments according to the current paradigm and should be signposts of the cosmic web in the distant universe. We present NIKA2 data that are a contribution to this endeavour due to serendipity.

In 2007, the field in the direction of the nearby star GJ526 was mapped in $1.2 \mathrm{~mm}$ continuum waveband using the MAMBO camera installed on the IRAM 30-meter radiotelescope at the time and revealed a quasi-alignment of the star and five compact sources in projection on the sky. It was not possible to decisively conclude whether these sources were background SMGs or a large, clumpy circumstellar disk seen edge-on around this star [15]. As cautiously stated in this paper, only future observations could definitely decide since these sources would stay fixed if in the background or move at the same rate and in the same direction as the star if clumps of a circumstellar disk. So a possible displacement as large as 22.6" in the south-west direction according to the star proper motion after ten years in 2017 motivated us to observe this field with the NIKA2 camera to determine the origin of these sources.

In this contribution, we report our finding that the five MAMBO sources did not move over this 10 year period and so they must be SMGs in the distant background beyond the star. It was argued in [15] that this was statistically unlikely in assuming a mean number surface density of SMGs but we are now led to believe that the GJ526 field presents instead 
an overdensity of SMGs. In this paper, we discuss the remarkable spatial distribution of these sources in the $1.15 \mathrm{~mm}$ NIKA2 map along a filament-like structure reminiscent of the cosmic web revealed in the cosmological hydrodynamical simulations.
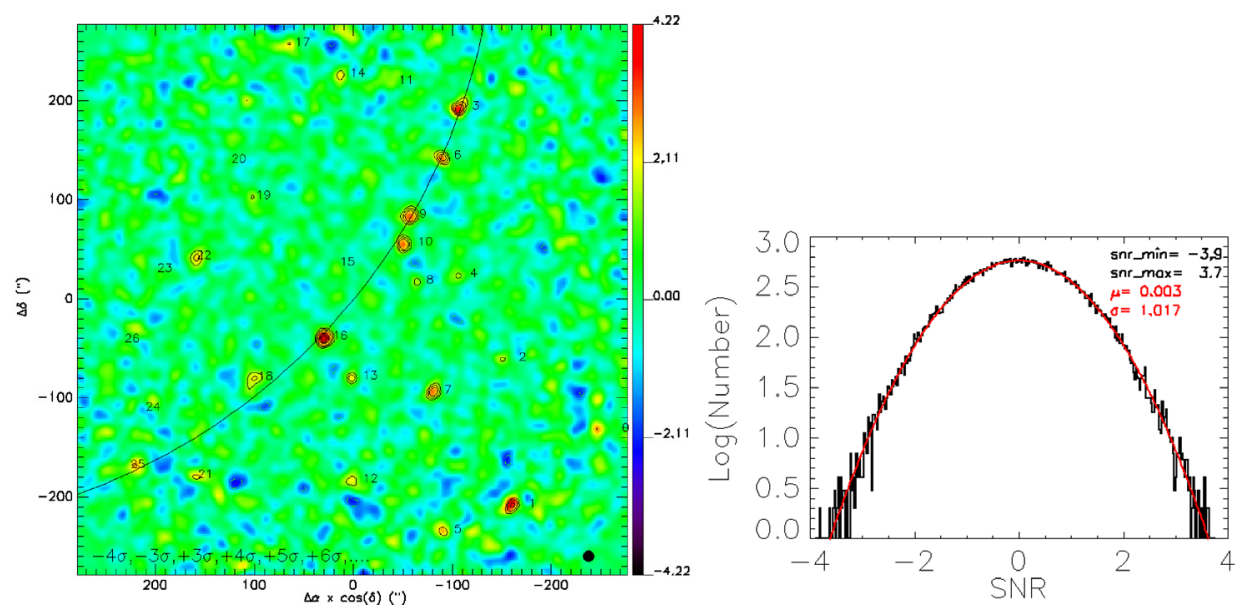

Figure 1. Left : GJ526 NIKA2 map at 1.15mm (color scale : mJy/beam, contours : signalto-noise ratio). Curve traces the filament-like structure identified across the map. Beam size is 11.2" (black filled circle at brc). Map center (J2000) : $13 \mathrm{~h} 45 \mathrm{~m} 44.52 \mathrm{~s}$ and $+14^{\circ} 53^{\prime} 20.6^{\prime \prime}$. Right : Signal-to-noise ratio histogramm of the jack-knifed map at $1.15 \mathrm{~mm}$ (in red, Gaussian distribution with zero mean and $\sigma=1$ for comparison).

\section{NIKA2 observations and the $1.15 \mathrm{~mm}$ map}

The NIKA2 observations were obtained during the science verification observations of the NIKA2 camera on 2017 april 24, 2018 february 14, 17, 18, 19, 20 and 2018 march 14, in average weather conditions (atmospheric opacity between 0.20 and 0.45 at $225 \mathrm{GHz}$ ) and at elevation between $33^{\circ}$ and $67^{\circ}$. The observing strategy consisted of a series of $9^{\prime} \times 4.5^{\prime}$ on-the-fly scans with a scanning speed of $40^{\prime} / \mathrm{sec}$ along two orthogonal orientations $\left(+60^{\circ}\right.$, $+150^{\circ}$ ) in the AZ-EL coordinate system at the telescope to minimise residual stripping patterns in the maps. These observations are split into 167 scans that correspond to a total of 9.3 hours of observations on source. The time ordered information (TOI) of each detector was processed in order to remove the contribution of atmospheric and electronic correlated noises and to obtain the maps at $1.15 \mathrm{~mm}$ and $2.0 \mathrm{~mm}$ following the procedure described in [16] and Ponthieu et al. (in preparation). Point sources are detected by matching the maps with a Gaussian Point-Spread-Function. Position accuracy is better than 3 arcseconds (for each coordinate) for sources above $4 \sigma$ and the absolute photometric accuracy is $\sim 15 \%$ [14].

In the limited space of this presentation, we shall discuss only the NIKA2 map at $1.15 \mathrm{~mm}$ and plan a complete presentation including the NIKA2 map at $2.0 \mathrm{~mm}$ in a forthcoming paper. We present the resulting $1.15 \mathrm{~mm}$ NIKA2 map in Fig. 1 and astrometry and photometry of the ten unambiguously detected sources with a signal-to-noise ratio (SNR) larger than 4 in Table 1. In order to determine this detection threshold, we have analysed the map noise in producing a jack-knifed map to compute SNRs in all pixels and found that their distribution are Gaussian, centered on zero and have a standard deviation of unity as expected for normal noise (see Fig. 1 right). In these conditions, no spurious sources with $S N R>4$ are statistically 
Table 1: NIKA2 astrometry and photometry of unambiguously detected sources at $1.15 \mathrm{~mm}$.

\begin{tabular}{rcccc}
\hline \hline $\begin{array}{r}\text { ID } \\
\mathrm{H} 3\end{array}$ & $\begin{array}{c}\alpha_{2000} \\
(\mathrm{~h} \mathrm{~m} \mathrm{~s})\end{array}$ & $\begin{array}{c}\delta_{2000} \\
\left({ }^{\circ}{ }^{\prime \prime}\right)\end{array}$ & $\begin{array}{c}S_{260 G H z} \\
(\mathrm{mJy})\end{array}$ & $\begin{array}{c}S N R \\
260 \mathrm{GHz}\end{array}$ \\
\hline \hline 1 & $13: 45: 33.48$ & $14: 49: 50.5$ & $3.96 \pm 0.95$ & 5.3 \\
3 & $13: 45: 37.18$ & $14: 56: 31.9$ & $3.67 \pm 0.81$ & 6.1 \\
6 & $13: 45: 38.36$ & $14: 55: 42.2$ & $2.95 \pm 0.68$ & 5.7 \\
7 & $13: 45: 38.97$ & $14: 51: 45.4$ & $2.87 \pm 0.63$ & 6.1 \\
9 & $13: 45: 40.61$ & $14: 54: 42.8$ & $3.13 \pm 0.65$ & 6.9 \\
10 & $13: 45: 41.09$ & $14: 54: 14.8$ & $2.97 \pm 0.62$ & 6.8 \\
13 & $13: 45: 44.69$ & $14: 51: 59.4$ & $2.08 \pm 0.53$ & 4.7 \\
16 & $13: 45: 46.63$ & $14: 52: 39.7$ & $4.11 \pm 0.75$ & 9.6 \\
18 & $13: 45: 51.51$ & $14: 51: 59.0$ & $2.15 \pm 0.57$ & 4.5 \\
22 & $13: 45: 55.51$ & $14: 54: 1.1$ & $2.40 \pm 0.63$ & 4.6 \\
\hline \hline
\end{tabular}

expected in the $500^{\prime \prime} \times 500^{\prime \prime}$ map owing to the number of independent beams $\left(11.2^{\prime \prime}\right.$ at $1.15 \mathrm{~mm}$ ) in it. There are also sixteen additional sources with $3<$ SNR $<4$ but three or four spurious positive sources are statistically expected at this significance level. Source IDs in Fig. 1 and Table 1 are assigned to all SNR $>3$ sources.

We found that four of the five MAMBO sources (see section GJ526 in Table 2 of [15]) have been detected anew in the NIKA2 map at $1.15 \mathrm{~mm}$ and are identified as ID 7, 9, 10, 16 in Table 1 herein. The fifth MAMBO source - MM134543+145317, a 4.3 $\sigma$ detection in [15] - has been searched in the $1.15 \mathrm{~mm}$ NIKA2 map and identified but only at a level of $2.8 \sigma$. Nonetheless, such a difference in SNR between the NIKA2 and MAMBO observations is statistically expected. As already mentionned in the introduction, our main finding is that all five MAMBO sources did not move between 2007 and 2017 and so they must be SMGs in the distant background.

The MAMBO flux densities given at $250 \mathrm{GHz}$ in Table 2 of [15] are comparable with the NIKA2 flux densities in Table 1 herein to better than 2.4 times the quadratically combined uncertainties of the two measurements. In Table 1, the flux density uncertainties include both statistical and systematic photometric uncertainties (this latter one is $\sim 15 \%$ [14]).

\section{Filament-like structure in the GJ526 field}

As stated above, there are ten sources unambiguously detected (SNR> 4) above $2 \mathrm{mJy}$ at $1.15 \mathrm{~mm}$ in the whole NIKA2 map. This can be usefully compared to the cumulative source number counts in other fields at a similar wavelength. To make this comparison meaningful, we shall restrict the map to its central region within a radial distance of $150^{\prime \prime}$ where the noise rms is uniform at the $10 \%$ level. In this region, there are six sources (ID 7, 9, 10, 13, 16, 18) that are unambiguously detected above $2 \mathrm{mJy}$ at $1.15 \mathrm{~mm}$. Combination of several single dish studies of the COSMOS field, the Lockman Hole North field and the GOODS field, provides the cumulative source number count $N\left(S_{1.2 \mathrm{~mm}}>2.0 \mathrm{mJy}\right)=500$ sources $/ \mathrm{deg}^{2}$ [17]. The Poisson probability that six sources be found within $r<150^{\prime \prime}$ given this mean surface number density is as low as $3.7 \%$. However, the GJ526 field we picked for our NIKA2 observations is one out of 50 similar-sized fields targeted in the initial MAMBO survey to search for circumstellar disks [15]. So this overdensity in the GJ526 field might very well be a statistical fluctuation in the whole MAMBO survey (note that no other MAMBO field 
shows such an overdensity). We turn now to the more intriguing spatial distribution of these SMGs along a filament-like structure.

It is remarkable that seven SMGs (IDs 3, 6, 9, 10, 16, 18, 25) are spatially distributed along a slightly curved filament-like structure in projection on the sky as highlighted in Fig. 1 with an arc (solid line). The first six sources are unambiguously detected and reported in Table 1 while the last one (ID 25) is only a possible $3.3 \sigma$ detection with a $1.15 \mathrm{~mm}$ flux density of $2.61 \pm 0.79 \mathrm{mJy}$. We can estimate the probability that such a quasi-alignment occurs in using the average source number surface density $N\left(S_{1.2 \mathrm{~mm}}>2.0 \mathrm{mJy}\right)=500$ sources/degree ${ }^{2}$, noted $\mu$ below, in small, consecutive sectors with a deviation angle $\delta$ from strict alignment of $\pm 10^{\circ}$ (see sketch in Fig. 2). Then, for a triplet of sources, the Poisson probability for the third source not to deviate more than $\delta$ from alignment with the first two sources is $\mu \times \pi r^{2} \times 2 \delta / 360^{\circ}$, where $r$ is the distance between the second and the third sources.

As sketched in Fig. 2, applying this for-

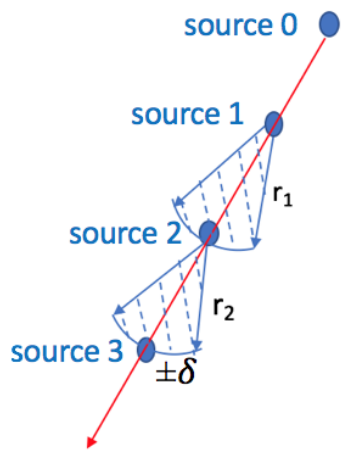
mula for the first triplet in the NIKA2 map (source ID's 3, 6, 9), the probability of occurence of quasi-alignement within $\pm 10^{\circ}$ is 500 sources $/ \mathrm{deg}^{2} \times \pi\left(70^{\prime \prime} / 3600^{\prime \prime}\right)^{2} \times$ $20^{\circ} / 360^{\circ}=3.3 \%$ where $r_{1}=70^{\prime \prime}$ is the distance between source IDs 6 and 9. Similarly, the probability is $0.6 \%$ for the second triplet (ID's $6,9,10)$ with $r_{2}=30^{\prime \prime}$, it is $11 \%$ for the third triplet (IDs $9,10,16$ ) with $r_{3}=130^{\prime \prime}$, it is $4.3 \%$ for the fourth triplet (ID's 10, 16, 18) with $r_{4}=80^{\prime \prime}$, and it is $15 \%$ for the fifth triplet (ID's 16, 18,

Figure 2. Estimation of chance alignment of sources.
25) with $r_{5}=150^{\prime \prime}$. The combined probability $\left(\prod_{i=1}^{5} \mu \pi r_{i}^{2} 2 \delta / 360^{\circ}\right)$ is then as low as $1.410^{-7}$ and so it is very unlikely that this filament-like structure has occured by chance, even in accounting for the fact that 50 similarsized fields have been observed in the initial MAMBO survey. So this apparent structure in the NIKA2 map is statistically significant.

This filament-like structure stretches across the whole map which is $500^{\prime \prime}$ in size. So, it extends at least over $\sim 4 \mathrm{cMpc}$ in using the scaling factor $8.3 \mathrm{kpc} /{ }^{\prime \prime}$ that does not depend strongly on redshift comprised between 1 and 6 in standard cosmology ${ }^{1}$. In addition, the angular separations $r_{i}$ specified above between these seven SMGs correspond to linear separations comprised between $\sim 0.25 \mathrm{cMpc}$ and $\sim 1.25 \mathrm{cMpc}$ in using the same redshift and scaling factor. This extent and these separations are comparable with the spatial distribution of dark matter haloes hosting galaxies in the high resolution cosmological simulation NewHorizon [19] for instance. This match between observations and simulations suggests that the structure in the NIKA2 map may be a cosmic filament. A complete presentation of this hypothesis and additional evidences, noticeably high luminosities $\left(\mathrm{L}_{F I R}>10^{12} \mathrm{~L}_{\odot}\right)$ and high star formation rates (SFR $>300 \mathrm{M}_{\odot} / \mathrm{yr}$ ) of the seven SMGs estimated with the $2.0 \mathrm{~mm}$ NIKA2 map and supplemental data from Herschel/SPIRE, will be published elsewhere.

\section{Conclusion}

In the $1.15 \mathrm{~mm}$ NIKA2 map of the GJ526 field presented here, there is an overdensity of SMGs characterized by an apparent filamentary structure in projection on the sky that is un-

\footnotetext{
${ }^{1}$ https://irsa.ipac.caltech.edu/Missions/herschel.html
} 
likely chance occurence and is visually reminiscent of a cosmic filament as cosmological simulations have shown (Illustris [18], NewHorizon [19], Uchuu [20]). However, confirmation of such a filament requires now measurements of spectroscopic redshifts of the milliJansky unlensed SMGs in using molecular and atomic lines with the most sensitive arrays such as NOEMA and ALMA. Also, comparison of our observed structure to the most recent cosmological simulations listed above is presently hampered by the fact they do not track specifically the rapidly star forming galaxies (main properties of SMGs). In fact, the less recent GADGET-2 simulation [21] was designed specifically to track massive galaxies with high star formation rates $\left(>280 \mathrm{M}_{\odot} / \mathrm{yr}\right)$ but the study does not present their spatial distribution. Specific tools to search cosmological simulations to better isolate SMGs are needed and complementary to the high-speed and deep mapping capability attained with NIKA2.

While galaxy surveys in the optical domain have already revealed the distinctive pattern of voids, sheets, filaments and nodes characterising the structure of the universe at low redshift, spatial distribution of SMGs have the potential to reveal similar structure but at high redshift.

\section{Acknowledgements}

This work is based on observations carried out during the science verification program of NIKA2 on the IRAM 30m telescope. IRAM is supported by INSU/CNRS (France), MPG (Germany) and IGN (Spain). We are grateful to the IRAM staff for their dedicated support. This research has made use of the SIMBAD database, operated at CDS, Strasbourg, France. This research has made use of the NASA/IPAC Extragalactic Database (NED), which is operated by the Jet Propulsion Laboratory, California Institute of Technology, under contract with the National Aeronautics and Space Administration.

\section{References}

[1] I. Smail, R.J. Ivison, A.W. Blain, ApJ 490, L5 (1997)

[2] D.H. Hughes, S. Serjeant, J. Dunlop et al., Nature 394, 241 (1998)

[3] M.L. Strandet, A. Weiss, J.D. Vieira et al., ApJ 822, 80 (2016)

[4] J.L. Puget, A. Abergel, J.P. Bernard et al., Astron. Astrophys. 308, L5 (1996)

[5] H. Dole, G. Lagache, J.L. Puget et al., Astron. Astrophys. 451, 417 (2006)

[6] J.R. Bond, L. Kofman, D. Pogosyan, Nature 380, 603 (1996)

[7] D. Kereš, N. Katz, D.H. Weinberg, R. Davé, MNRAS 363, 2 (2005)

[8] A. Dekel, Y. Birnboim, G. Engel et al., Nature 457, 451 (2009)

[9] A. Monfardini, A. Benoit, A. Bideaud et al., ApJS 194, 24 (2011)

[10] A. Catalano et al., Astron. Astrophys. 569, A9 (2014)

[11] M. Calvo et al., Journal of Low Temperature Physics, 184, 816 (2016)

[12] O. Bourrion et al., Journal of Instrumentation, 11, 11001 (2016)

[13] R. Adam et al., Astron. Astrophys. 609, A115 (2018)

[14] L. Perotto, N. Ponthieu, J.-F. Macías-Pérez et al., Astron. Astrophys. 637, A71 (2020)

[15] J.F. Lestrade, M.C. Wyatt, F. Bertoldi et al., Astron. Astrophys. 506, 1455 (2009)

[16] R. Adam, B. Comis, J.F. Macías-Pérez et al., Astron. Astrophys. 569, A66 (2014)

[17] R..R. Lindner, A.J. Baker, A. Omont et al., ApJ 737, 83 (2011)

[18] V. Springel, R. Pakmor, A. Pillepich et al., MNRAS, 475, 676 (2018)

[19] Y. Dubois, R. Beckmann, F. Bournaud et al., Astron. Astrophys. 651, A109 (2021)

[20] T. Ishiyama, F. Prada, A. Klypin et al., MNRAS 506, 4210 (2021)

[21] R. Davé, B.D. Oppenheimer, N. Katz et al., MNRAS, 408, 2051 (2010) 